\title{
Determinación de la composición fitoquímica del extracto hidroalcohólico de Zingiber officinale (kion) en la selva central del Perú
}

\author{
Determination of the phytochemical composition of the hydro-alcoholic extract of Zingiber officinale \\ (ginger) in the central jungle of Peru
}

\begin{abstract}
Diana Esmeralda Andamayo Flores ${ }^{1, a}$, Venancio Santiago Navarro Rodriguez ${ }^{1, a}$, Diana Esmeralda Castillo Andamayo ${ }^{1, a}$, Vilma Amparo Junchaya Yllescas ${ }^{1, b}$, Rosario Mercedes Chuquillanqui Galarza ${ }^{1, b}$
\end{abstract}

\section{RESUMEN}

Objetivos: Determinar la composición fitoquímica del extracto hidroalcohólico del Zingiber officinale "Kion" de la Selva Central del Perú. Material y Métodos: Se utilizó básicamente el método científico de observación estructurada, siendo una investigación de tipo básica, nivel descriptivo de diseño no experimental, observacional descriptivo de corte transversal. El diseño que se empleó responde al de una investigación por objetivos. Resultados: La muestra vegetal ha sido estudiada y clasificada en el laboratorio de la Universidad Nacional Mayor de San Marcos, se identificó a la especie botánica según el sistema de clasificación de cronquist (1988) definiendo como clase: liliopsida, sub clase: zingiberidae, orden: zingiberales, familia: zingiberaceae, género: Zingiber, especie: Zingiber officinale Roscoe, nombre vulgar: "kion". De acuerdo a la identificación de metabolitos secundarios se identificó como (+++) Reacción muy evidente; (++) Reacción evidente; (+) Reacción poco

evidente; (-) No hubo reacción. Conclusiones: De acuerdo a la identificación de metabolitos secundarios, la especie Zingiber officinale Roscoe, presenta antocianinas, alcaloides, flavonoides, aminoácidos, saponinas, taninos, azúcares reductores y fenoles, no encontrándose en la muestra lactonas, cardenólidos, esteroides, triterpenos ni quinonas. De acuerdo a los resultados tenemos en primer lugar a los alcaloides con una reacción muy evidente seguido de los aminoácidos con una reacción evidente, teniendo similitud con la información reportada por otros autores.

PALABRAS CLAVES: fitoquímica, extracto hidroalcohólico, Zingiber officinale Roscoe.

\section{SUMMARY}

Objectives: Determine the phytochemical composition of the hydro alcoholic extract of the Zingiber officinale "Ginger" of the Central Jungle of Peru. Material and Methods: The scientific method of structured observation was basically used, being a basic type research, descriptive level of non-experimental design, descriptive of cross section. The design that was used responds to that of an investigation by objectives. Results: The plant sample has been studied and classified in the laboratory of the National University of San Marcos, the botanical species was identified according to the classification system of cronquist (1988) defining as class: liliopsida, sub class: zingiberidae, order: zingiberales, family: zingiberaceae, genus: Zingiber, species: Zingiber officinale Roscoe, vulgar name: "kion". According to the identification of secondary metabolites, it was identified as (+++) Very evident reaction; (++) Obvious reaction; (+) Less evident reaction; (-) There was no reaction. Conclusions: According to the identification of secondary metabolites, the species Zingiber officinale Roscoe presents anthocyanins, alkaloids, flavonoids, amino acids, saponins, tannins, reducing sugars and phenols, not found in the sample lactones, 
cardenolides, steroids, triterpenes or quinones. According to the results we have in the first place the alkaloids with a very evident reaction followed by the amino acids with an evident reaction, having similarity with the information reported by other authors.

KEYWORDS: phytochemistry, hydro alcoholic extract, Zingiber officinale Roscoe.

\section{INTRODUCCIÓN}

En nuestro país encontramos diferentes regiones, una de ellas la Andina; que cuenta con una gran variedad de recursos naturales (plantas medicinales), que ayuda a combatir diversas enfermedades que aquejan a todos los seres humanos, sin distinción de edad, sexo o condición económica.

El jengibre tiene un sabor picante, similar al del ajo, pero a diferencia de éste, no genera intolerancia a nivel digestivo. Las raíces viejas y secas tienen un sabor más fuerte. El jengibre es analgésico y antipirético, lo que reduce el dolor en personas con síntomas o que padecen enfermedades inflamatorias. Asimismo, tiene un efecto antioxidante anulando la actividad de radicales libres, causantes de daño y envejecimiento celular precoz que contribuyen al desarrollo enfermedades como cáncer, diabetes, infartos, manchas en la piel, entre otras patologías. El efecto antitusivo y descongestivo del jengibre actúa sobre el sistema respiratorio disminuyendo en tiempo y forma la congestión y la tos; del mismo modo, ayuda en la motilidad gastrointestinal; asimismo, disminuye los síntomas estomacales y menstruales (1).

En la actualidad los medicamentos han revolucionado la terapéutica moderna y han salvado cantidad de vidas de niños y adultos, y seguirán siendo útiles, cuando son bien utilizadas. Sin embargo, la ingestión de cualquier sustancia medicamentosa debe ser vigilada y recetada por un profesional entrenado en ese menester, y se debe procurar que así sea, pues nuestra salud se lo merece (2).

Por lo antes mencionado, fue necesario determinar la composición fitoquímica del extracto hidroalcohólico del Zingiber officinale "Kion" de la Selva Central del Perú con la finalidad de obtener información relevante respecto a los principios activos de la misma con fines terapéuticos y otros.

\section{MATERIAL Y MÉTODOS}

- Vaso de precipitación de $250 \mathrm{ml}$.

- Probeta de $100 \mathrm{ml}$.
- Cocinilla eléctrica.

- Cronómetro

- Probeta de $500 \mathrm{ml}$.

- Equipo de baño Maria.

En la investigación se aplicó básicamente el método científico: observación estructurada (3).

La población de la investigación fue la planta de Zingiber officinale "kion".

La muestra considerada en la investigación como unidad de análisis es la raíz de Zingiber officinale "Kion".

Técnicas e instrumentos de recolección de datos y de procesamiento de la investigación (4).

\section{Recolección de datos}

Para realizar la evaluación de la taxonomía de Zingiber officinale "Kion", se llevó la planta al Museo de Historia Natural de la Universidad Nacional Mayor de San Marcos para la respectiva clasificación taxonómica e identificación de la planta.

\section{Tratamiento de la muestra vegetal}

Las raíces de Zingiber officinale "Kion", fueron recolectadas de aquellas plantas que crecen en la jurisdicción de la Selva Central de Perú después de concluir con la floración de la especie.

La muestra respectiva se trasladó al Laboratorio de Química Orgánica de la Universidad Privada de Huancayo "Franklin Roosevelt", donde fue exudada y deshidratada a temperatura del medio ambiente, obteniéndose la unidad de muestra luego de la maceración en el extracto hidroalcohólico por espacio de 10 días.

\section{Determinación de los componentes fitoquímicos}

Se realizó la marcha fitoquímica para obtener los diferentes ensayos con reacciones químicas de identificación mediante cambios de color o formación de precipitación para determinar la presencia de metabolitos secundarios, tales como: flavonoides (ensayo de Shinoda), compuestos fenólicos (cloruro férrico), alcaloides (ensayo de Hager, Draggendorff, Mayer y Wagner), triterpenos y esteroides (ensayo de Liebermann-Buchard), quinonas (ensayo de Bortranger), compuestos lactónicos y cumarinas 
(ensayo de Baljet), antocianidinas (ensayo de Rosenhein), azúcares reductores (ensayo de Fehling) y saponinas (ensayo de Molisch).

\section{Quinta Fase}

A la fase acuosa total se agregó $25 \mathrm{ml}$. de hexano formándose 2 fases, la fase orgánica y la fase acuosa, a la fase orgánica se agregó sulfato de sodio anhidro para deshidratar, la cual fue sometida a reacciones de identificación de flavonoides, esteroides, catequinas, alcaloides, cardenólidos y leucoantocianidinas; la fase acuosa se unió a la fase acuosa total, obteniéndose una nueva muestra la cual será sometida a reacciones de identificación de flavonoides y leucoantocianidinas.

\section{Sexta Fase}

A un mililitro de muestra se le agregó $50 \mathrm{ml}$. de agua, llevándolo luego a baño maría durante 15 minutos. Finalmente se filtró en caliente obteniéndose la otra muestra la cual fue sometida a reacciones de identificación de saponinas.

\section{RESULTADOS}

\section{Resultado de muestra vegetal}

La muestra vegetal fue estudiada y clasificada en el laboratorio de la Universidad Nacional Mayor de San Marcos, según el Sistema de Clasificación de Cronquist (1988).

Muestra vegetal clasificada como: Zingiber officinale

\section{DISCUSIÓN}

\section{De la clasificación de la especie botánica}

En la tabla 1 se muestra la clasificación de la especie botánica como, especie: Zingiber officinale Roscoe con una posición taxónómica, división: magnoliophyta;

Tabla 1. Identificación de la especie botánica

\begin{tabular}{ll}
\hline DIVISION & MAGNOLIOPHYTA \\
CLASE & LILIOPSIDA SUB \\
CLASE & ZINGIBERIDAE \\
ORDEN & ZINGIBERALES \\
FAMILIA & ZINGIBERACEAE \\
GENERO & Zingiber \\
ESPECIE & Zingiber officinale Roscoe \\
Nombre Vulgar & "kión" \\
Determinado por Mg. Marioa Isabel La Torre \\
Lima, 18 de octubre del 2017
\end{tabular}

clase liliopsida: sub clase zingiberidae; orden: Zingiberales; familia: zingiberaceae: genero: Zingiber. Según Enriquez y Yachachin se muestra diferencia en la división: angioesperma, y clase: monocotiledónea coincidiendo en el resto la clasificación taxonómica $(5,6)$.

\section{De los resultados de los grupos fitoquímicos}

La muestra vegetal fue analizada en el Centro De Control Analítico del Instituto de Investigación De Recursos Naturales, según Orden de análisis: 0001 En la tabla 2 según protocolo de análisis $\mathrm{N}^{\circ} 00193$ CPF-2018. Se determinó a través de una reacción cualitativa el metabolito Antocianinas dando un resultado $(+)$, que significa una reacción poco evidente, los autores en comparación no hicieron el análisis del metabolito en mención. Respecto a este metabolito nos indica una mínima presencia de Antocianinas en la muestra. $(5,6,7)$.

En el análisis de Alcaloides con el ensayo de Dragendorff dio como resultado $(+++)$, indicando una reacción muy evidente, es decir con presencia considerable de este metabolito, según los análisis de Yachachin e Inca, muestran resultado negativo (-) (6, $7)$; mientras que Enriquez muestra un resultado $(+)$ que indica presencia de este metabolito (5).

En el análisis de Lactonas según el ensayo de Baljet nos muestra un resultado (-), que nos indica que no hubo reacción, es decir ausencia de este metabolito. Según Enriquez y Yachachin, nos indica un resultado $(++)$, que indica una reacción evidente $(5,6)$; mientras que Inca no realizó el análisis de este metabolito (7).

En el análisis de Flavonoides según el ensayo de Shinoda nos muestra un resultado $(+)$, que nos indica una reacción poco evidente, es decir una baja presencia de este metabolito. Según Enriquez, Yachachin, e Inca, nos indica un resultado $(++)$, que indica una reacción evidente, es decir presencia de metabolito $(5,6,7)$.

En el análisis de Aminoácidos según el ensayo de Reacción Xantoproteica y reacción de acetato de plomo alcalino nos muestra un resultado (++), que nos indica una reacción evidente, es decir presencia considerable de este metabolito. Según Enriquez e Inca, no indica un resultado $(+++)$, que indica una reacción muy evidente $(5,7)$; mientras que Yachachin no realizó el análisis de este metabolito, lo cual no indica que existe considerable presencia de aminoácidos en la muestra (6). 
Tabla 2. Identificación de metabolitos secundarios.

\begin{tabular}{|c|c|c|c|}
\hline \multicolumn{4}{|c|}{ MARCHA FITOQUÍMICA } \\
\hline METABOLICO & ENSAYO & MËTODOS & RESULTADOS \\
\hline \multirow[t]{2}{*}{ ANTOCIANINAS } & Reacción Cualitativa & Cualitativo & + \\
\hline & Reaccion de Dragendorff & Cualitativo & +++ \\
\hline \multirow[t]{2}{*}{ ALCALOIDES } & Reacción de Mayer & Cualitativo & + \\
\hline & Reacción deWagner & Cualitativo & - \\
\hline LACTONAS & Reacción de Baljet & Cualitativo & - \\
\hline FLAVONOIDES & Reacción Shinoda & Cualitativo & + \\
\hline AMINOACIOS & Reacción Xantoproteica & Cualitativo & ++ \\
\hline AMINOACIOS & $\begin{array}{l}\text { Reacción de acetano de plomo } \\
\text { alcalino }\end{array}$ & Cualitativo & ++ \\
\hline CARDENÓLODOS & Reacción de Kedde & Cualitativo & - \\
\hline ESTEROIDES & Reacción de Liebermann-Burchard & Cualitativo & - \\
\hline SAPONINAS & Reacción de espuma & Cualitativo & + \\
\hline TANINOS & Reacción con cloruo férrico & Cualitativo & + \\
\hline TRITERPENOS & Reacción de Liebermann-Burchard & Cualitativo & - \\
\hline QUINONAS & Reacción de Bomtrager & Cualitativo & - \\
\hline $\begin{array}{l}\text { AZUCATES } \\
\text { REDUCTORES }\end{array}$ & Reacción de Fehling & Cualitativo & + \\
\hline FENOLES & Reacción de cloruro férrico & Cualitativo & + \\
\hline
\end{tabular}

Leyenda:

+++ Reacción muy evidente

++ Reacción evidente

+ Reacción poco evidente

- No hubo reacción

En el análisis de Esteroides según el ensayo de Reacción de Liebermann Burchard nos muestra un resultado (-), que nos indica que no hubo reacción, es decir la no presencia de este metabolito. Los autores Enriquez, Yachachin e Inca no realizaron el análisis de este metabolito $(5,6,7)$.

En el análisis de Saponinas existe resultados diferentes, pero con presencias de este metabolito, según el ensayo de Reacción de espuma nos muestra un resultado $(+)$, que nos indica una reacción poco evidente, es decir una baja presencia de este metabolito. Según Enriquez y Yachachin, nos muestra un resultado (-) indicando la ausencia de este metabolito $(5,6)$; según Inca nos indica un resultado $(++)$, que indica una reacción evidente, es decir presencia considerable de este metabolito (7). De acuerdo a nuestro resultado y considerando a uno de los autores se tiene presencia de este metabolito.

En el análisis de Taninos, según el ensayo de Reacción con cloruro férrico nos muestra un resultado $(+)$, que nos indica una reacción poco evidente, es decir una baja presencia de este metabolito. Según Enriquez nos muestra un resultado $(+)$ indicando baja presencia de este metabolito (5); según Inca nos indica un resultado (++), que indica una reacción evidente, es decir presencia considerable de este metabolito; Inca no realizó el análisis de este metabolito (7).

En el análisis de Triterpenos según el ensayo de Reacción Liebermann - Burchard nos muestra un resultado (-), que nos indica que no hubo reacción, es decir ausencia de este metabolito. Según Enriquez, Yachachin e Inca de la misma manera un resultado (-), que indica que no hubo reacción, es ausencia de este metabolito $(5,6,7)$.

En el análisis de Quinonas según el ensayo de Reacción de Borntrager nos muestra un resultado (-), que nos indica que no hubo reacción, es decir no existe presencia de este metabolito. Según Yachachin, el resultado es (-) indicando que no hubo reacción (6); Enriquez con el mismo ensayo encontró un resultado $(+++)$ encontrado una reacción muy evidente es decir con alto contenido de quinonas, esta diferencia se 
debe especialmente a la procedencia de la muestra, en nuestro caso nuestra muestra corresponde al producto cultivado en Pichanaki (5); mientras que Inca no realizó el análisis de este metabolito (7).

En el análisis de Azúcares Reductores según el ensayo de Reacción de Fehling nos muestra un resultado $(+)$, que nos indica una reacción poco evidente, es decir una mínima presencia de este metabolito. Según Yachachin, el resultado es $(++)$ que indica una reacción evidente, es decir presencia considerable de este metabolito (6); Enriquez con el mismo ensayo encontró un resultado $(+)$ encontrado una reacción poca evidente es decir con un mínimo contenido de azúcares reductores (5); según Inca, se encontró un resultado $(+++)$ que indica una reacción muy evidente, es decir con un alto contenido de azúcares reductores (7).

En el análisis de Fenoles según el ensayo de Reacción de cloruro férrico nos muestra un resultado $(+)$, que nos indica una reacción poco evidente, es decir una mínima presencia de este metabolito. Según Yachachin, el resultado es $(++)$ que indica una reacción evidente, es decir, presencia considerable de este metabolito (6); Enriquez e Inca no realizaron el análisis de este metabolito $(5,7)$.

Las diferencias encontradas se deben especialmente a la procedencia de la muestra, en nuestro caso la muestra corresponde al producto cultivado en el distrito de Pichanaki, Provincia de Chanchamayo.

\section{CONCLUSIONES}

1. Se identificó a la especie botánica según el sistema de clasificación de cronquist (1988) definiendo como clase: liliopsida, sub clase: zingiberidae, orden: zingiberales, familia: zingiberaceae, género: Zingiber, especie: Zingiber officinale Roscoe, nombre vulgar: "kion"; teniendo similitud con la información reportada por otros autores.

2. De acuerdo a la identificación de metabolitos secundarios, la especie Zingiber officinale Roscoe, presenta antocianinas, alcaloides, flavonoides, aminoácidos, saponinas, taninos, azúcares reductores y fenoles, no encontrándose en la muestra lactonas, cardenólidos, esteroides, triterpenos ni quinonas. De acuerdo a los resultados tenemos en primer lugar a los alcaloides con una reacción muy evidente seguido de los aminoácidos con una reacción evidente.

\section{Correspondencia:}

Dra. Diana Esmeralda Andamayo Flores

Correo electrònico: dandamayo@uroosevelt.edu.pe

\section{REFERENCIAS BIBLIOGRÁFICAS}

1. Ministerio de Producción y trabajo. Jengibre. Nutrición y educación alimentaria. 2017;60: 1-4.. (Citado el 29 de setiembre del 2017) Disponible en: http:// www.alimentosargentinos.gob.ar/ HomeAlimentos/Nutricion/fichaspdf/Ficha_60_ Jengibre.pdf

2. Armero L, Cruz S. Factores que inciden sobre la automedicación en las personas que habitan en la vereda los llanos del Municipio de Popayan con edades respectivas entre 18 y 85 años. Tesis de Grado. Popayán: Universidad Nacional Abierta y a Distancia; 2011.

3. Hernández R, Fernández C, Baptista P. Metodología de la Investigación. Ciudad de México: Edit. McGraw-Hill; 2006.

4. Valderrama S. Pasos para elaborar Proyectos y Tesis de Investigación científica. Lima: Edit. San Marcos EIRL; 2010.

5. Enriquez F, Prieto V. Estudio farmacognóstico y fitoquímico del rizoma de Zingiber officinale roscoe "Jengibre" de la ciudad de Chanchamayo Región Junín - Perú. Tesis de Grado. Trujillo: Universidad Nacional de Trujillo;2007.

6. Yachachin E. Caracterización fisicoquímica del extracto expectorante de ajo (Allium sativum L.), kión (Zingiber officinale L.) eucalipto (Eucaliptus globulus L.) y linaza (Linum usitatissimum L.) Tesis de Grado. Tarma, Perú: Universidad Nacional del Centro; 2013.

7. Inca T. Elaboración de un fitofármaco semisólido de acción adelgazante con diferentes dosis a base de alcachofa (Cynara cardunculus var scolymus), jengibre (Zingiber officinale) y cáscara de naranja (Citrus sinensis) administrado a personas para comparar su eficacia. Tesis de Grado. Riobamba, Ecuador: Escuela Superior Politécnica de Chimborazo; 2012.

Recibido: 12/10/2019

Aceptado: 18/01/2020 